\title{
Observations sur l'anisotropie hydraulique lors de la reconnaissance et de la construction de stockages souterrains de GPL en cavités minées
}

\author{
Observations of hydraulic anisotropy during the exploration \\ and construction of underground storages of LPG in mined caverns
}

\author{
E. AMANTINI, P. GENESTE \\ GÉOSTOCK*
}

Rev. Franç. Géotech. n 59 , pp. 43-47 (avril 1992)

\section{Résumé}

Des phénomènes d'anisotropie de perméabilité ont été mis en évidence lors de la reconnaissance et de la construction de certains stockages souterrains de GPL (Gaz de Pétrole Liquéfiés) en cavité minée.

L'étanchéité hydraulique des stockages souterrains repose sur l'existence d'un écoulement dirigé vers l'intérieur de la cavité en tout point de sa paroi et à tout instant de son exploitation. La connaissance de la géométrie des écoulements est donc essentiellement au dimensionnement des projets de stockage de GPL : répartition spatiale des perméabilités, position des conditions aux limites.

L'hétérogénéité des milieux reconnus est souvent à l'origine de l'anisotropie de perméabilité qui se manifeste par une anisotropie dans la distribution des écoulements ; il peut s'agir d'hétérogénéité lithologique ou due à la fissuration.

Les phénomènes d'anisotropie hydraulique sont mis en évidence et quantifiés par des essais hydrogéologiques appropriés d'interférences verticale et horizontale entre forages.

Pour les stockages en cavités minées, l'influence de l'anisotropie de perméabilité des milieux étudiés est un facteur particulièrement important pour le dimensionnement et la conception. Des conditions défavorables d'anisotropie, par exemple un fort contraste $\mathrm{Kh}>\mathrm{Kv}$, peuvent amener à augmenter la distance entre unités, voire à réaliser des dispositifs artificiels afin de minimiser les effets des interférences hydrauliques horizontales (rideaux d'eau verticaux). Un tel contraste au niveau des galeries peut également introduire l'existence de phénomènes transitoires, imposant la définition et le respect strict de règles d'exploitation limitant notamment les vitesses de montée en pression du stockage.

\section{Abstract}

Certain phenomena of anisotropy in rock permeability have been highlighted during the exploration and the construction of some underground storages of LPG (Liquefied Petroleum Gas) in mined cavern.

The hydraulic tightness of the underground storages is based on the existence of a flow directed towards the cavern at every point of the wall during the whole duration of its operation. The geometric knowledge of the flow is essential in order to design LPG storage projects : spatial repartition of the permeabilities, position of the flow boundaries.

The heterogeneity of the explored medium is often responsible for the anisotropy of the permeability, which leads to an anisotropy of the flow; the origin of the anisotropy can be lithologic, or due to the fissuration.

The phenomena of anisotropy are detected and quantified by appropriate hydrogeological horizontal and vertical interference tests between boreholes.

The influence of the anisotropy in the rock permeability of the considered medium is important when considering the design and dimensioning of the storages in mined caverns. Some unfavourable conditions of anisotropy, for instance a high variation $\mathrm{Kh}>\mathrm{Kv}$, can lead to an increase in the distance between two units, and in some cases the need to include artificial devices in order to minimize the effects of hydraulic horizontal interferences (vertical water curtain). Such a variation occurring at cavern level can also be responsible for transient phenomena, leading to the implementation of strict operating rules, limiting the maximum rate of pressure rise in the cavern.

- GÉOSTOCK, 7, rue E. et A.-Peugeot, 92563 Rueil-Malmaison (France). 


\section{RAPPEL DU PRINCIPE D'ÉTANCHÉITÉ HYDRAULIQUE APPLIQUÉ PAR GÉOSTOCK}

Le stockage souterrain en cavité minée concerne notamment des produits issus du raffinage (GPL ou Gaz de Pétrole Liquéfiés) qui sont stockés à un état de pression correspondant à leur tension de vapeur pour laquelle coexistent les phases liquide et vapeur à une température dépendant des conditions géothermiques locales et des conditions d'exploitation.

Le confinement de ces produits est assuré par l'existence, en tout point de la paroi de la cavité, d'un gradient de potentiel hydraulique dirigé vers l'intérieur. Ce critère suppose, qu'à tout instant de l'exploitation, le potentiel hydraulique de la roche hôte au voisinage du stockage soit supérieur à celui de la cavité majoré de coefficients de sécurité.

L'application de ce principe suppose la permanence des écoulements entre la nappe et le stockage, donc une bonne connaissance de la géométrie des écoulements et des conditions aux limites.

A titre d'exemple, pour un propane commercial dont la tension de vapeur à $20^{\circ} \mathrm{C}$ est de $0,75 \mathrm{MPa}$ relatif, les études menées par GÉOSTOCK montrent que la profondeur d'implantation requise pour le toit de la cavité serait de l'ordre de 100 à $120 \mathrm{~m}$ selon les conditions hydrogéologiques locales et la valeur du terme de sécurité pris en compte, soit largement supérieure à $75 \mathrm{~m}$ dans l'hypothèse d'un niveau phréatique proche de la surface.

Afin de s'affranchir d'éventuelles variations du niveau piézoméétrique, il est parfois nécessaire d'avoir recours à des ouvrages artificiels constitués par des forages disposés dans un plan horizontal au-dessus des cavités (les rideaux d'eau) ; maintenus à un potentiel hydraulique constant, ils contribuent à assurer la permanence des écoulements vers le stockage.

La connaissance du contexte hydrogéologique est donc primordiale : répartition verticale des perméabilités, continuité latérale des horizons aquifères susceptibles d'occassionner des écoulements vers le stockage, identification des conditions aux limites (supérieure. inférieure et latérale).

\section{EXEMPLES D'ANISOTROPIES DE PERMÉABILITÉ RENCONTRÉES}

La reconnaissance du contexte hydrogéologique d'un projet de stockage de GPL impose la définition d'une échelle d'investigation appropriée. Le volume de roche reconnu est généralement d'environ $500 \times 500 \times$ $200 \mathrm{~m}$, pour un volume de cavité de l'ordre de $200000 \mathrm{~m}^{3}$, le nombre de forages réalisés variant de 5 à 15 .

Les contextes géologiques reconnus par GÉOSTOCK lors de la reconnaissance de sites en vue du stockage des GPL en cavité minée sont diversifiés:

- milieux sédimentaires : craie, calcaires, grès, shales ;

- milieux fissurés : schistes, roches métasédimentaires, gneiss, granite.
Les perméabilités rencontrées sont généralement comprises dans une gamme allant d'environ $10^{-5} \mathrm{~m} / \mathrm{s}$ à $10^{-11} \mathrm{~m} / \mathrm{s}$.

Différents cas de contraste d'anisotropie de perméabilité ont été mis en évidence :

- $\mathrm{Kv}>\mathrm{Kh}$ : roches fissurées de type granite, gneiss ou roches métasédimentaires présentant des joints verticaux prédominants :

- Kh > Kv : cas de roches hétérogènes lithologiquement souvent d'origine sédimentaire (craie, shales, flyschs), l'anisotropie de perméabilité étant souvent due à la présence d'intercalations de niveaux subhorizontaux moins perméables.

$\mathrm{Kv}$ : composante verticale du tenseur de perméabilité ; Kh : composante horizontale du tenseur de perméabilité.

\section{MISE EN ÉVIDENCE IN SITU}

\subsection{Types d'essais mis en œuvre}

Les essais hydrogéologiques couramment mis en œuvre lors des phases de reconnaissance consistent en plusieurs types d'essais réalisés en continu lors de l'avancement des foragaes :

- essais d'injectivité de type Lugeon, supposant l'établissement d'un régime permanent radial-circulaire entre le puits et la couche testée; la zone faisant l'objet d'investigations est généralement réduite ;

- essais transitoires d'injection relaxation permettant de déterminer la transmissivité et le coefficient de peau $(\alpha$ skin $x)$ (modification des caractéristiques de la roche au voisinage du forage due à la foration);

- essais d'injection et/ou de production de plus longue durée permettant de mettre en évidence d'évenutelles hétérogénéités ou la présence de conditions aux limites.

Ces types de test permettent de mesurer la composante horizontale $(\mathrm{Kh})$ du tenseur de perméabilité au voisinage proche du forage.

Les essais d'interférence entre forages permettent de déterminer $\mathrm{Kh}$ à une échelle plus importante (de quelques mètres à plusieurs centaines de mètres).

Les essais d'interférence verticale sont adaptés à la mesure de la composante verticale du tenseur de perméabilité (Kv) et du rapport d'anisotropie (Kv/Kh). Ces essais consistent en la mesure de variation de pression sur un ou plusieurs forages observateurs, induite à partir d'un forage injecteur (ou producteur) situé à une cote différente.

L'interprétation est effectuée en régime transitoire par calage des valeurs mesurées sur une loi théorique (fonction de Theis), avec la prise en compte d'une éventuelle influence des conditions aux limites, permettant ainsi de déterminer $\mathrm{Kv}$.

\subsection{Exemple (Corée du Sud)}

Les résultats présentés concernent des essais d'interférence réalisés dans un contexte de gneiss fissurés dont la fissuration verticale induite une anisotropie importante de perméabilité. La figure 1 montre le dispositif utilisé permettant de tester la perméabilité d'un 
linéament mis en évidence par reconnaissance géophysique :

- l'essai entre BL - 1 et T - 1 permet de mesurer la perméabilité verticale au sein du joint, $\mathrm{Kv}=1,3 \cdot 10^{-8} \mathrm{~m} / \mathrm{s}$ (fig. 2 ) ;

- l'essai entre BL - 1 et $\mathrm{BL}$ - 5 permet de mesurer la perméabilité horizontale au sein du joint, $\mathrm{Kh}=3,3 \cdot 10^{-9} \mathrm{~m} / \mathrm{s}$, soit $\mathrm{Kv} / \mathrm{Kh}=4$ (fig. 3):

- les mesures sur $\mathrm{BH}-3$, situé en dehors de la zone fissurée, indiquent l'absence d'interférence, ce qui démontre l'existence d'une très faible perméabilité de la roche en l'absence de fissuration (fig. 4).

On notera que les interprétations réalisées prennent en compte l'influence d'une condition à la limite supé-

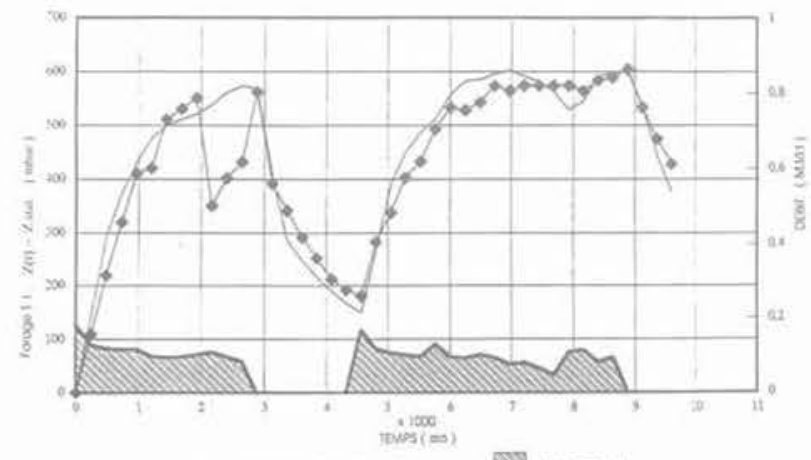

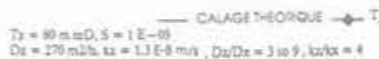

Fig. 2. - Interférence de BL-1 vers T-1,

Fig. 2. - interference test from BL-1 to T-1

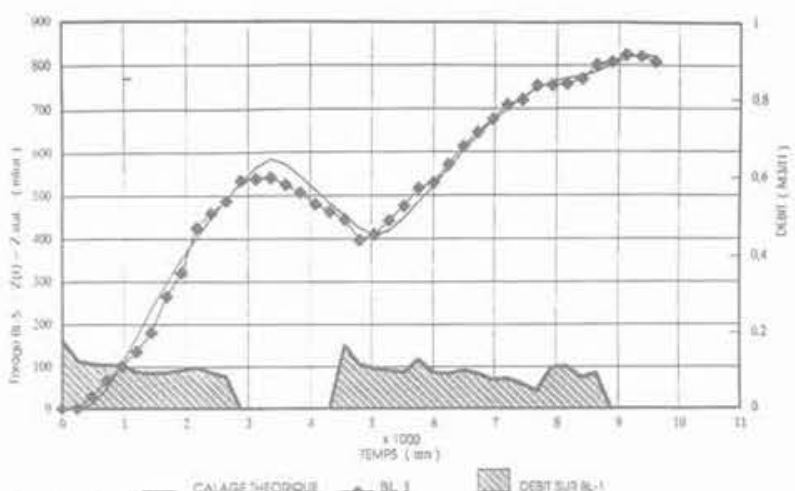

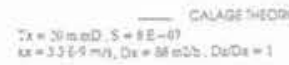

Fig. 3. - Interférence de $B L-1$ vers $B L-5$.

Fig. 3. - Interference test from $B L-1$ to $B L-5$.

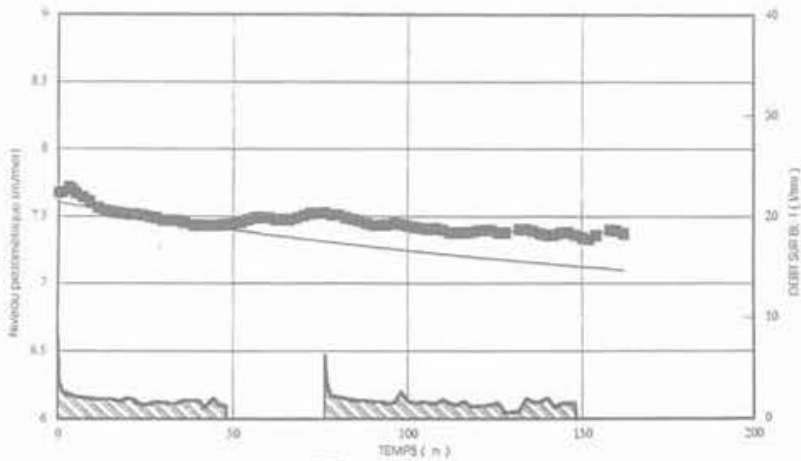

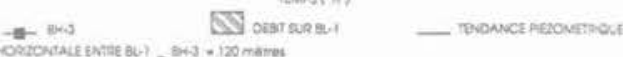

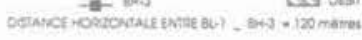

Fig, 4. - Evolution du niveau piézométrique de BH-3.

Fig. 4. - Evolution of the piezometric level of BH-3.

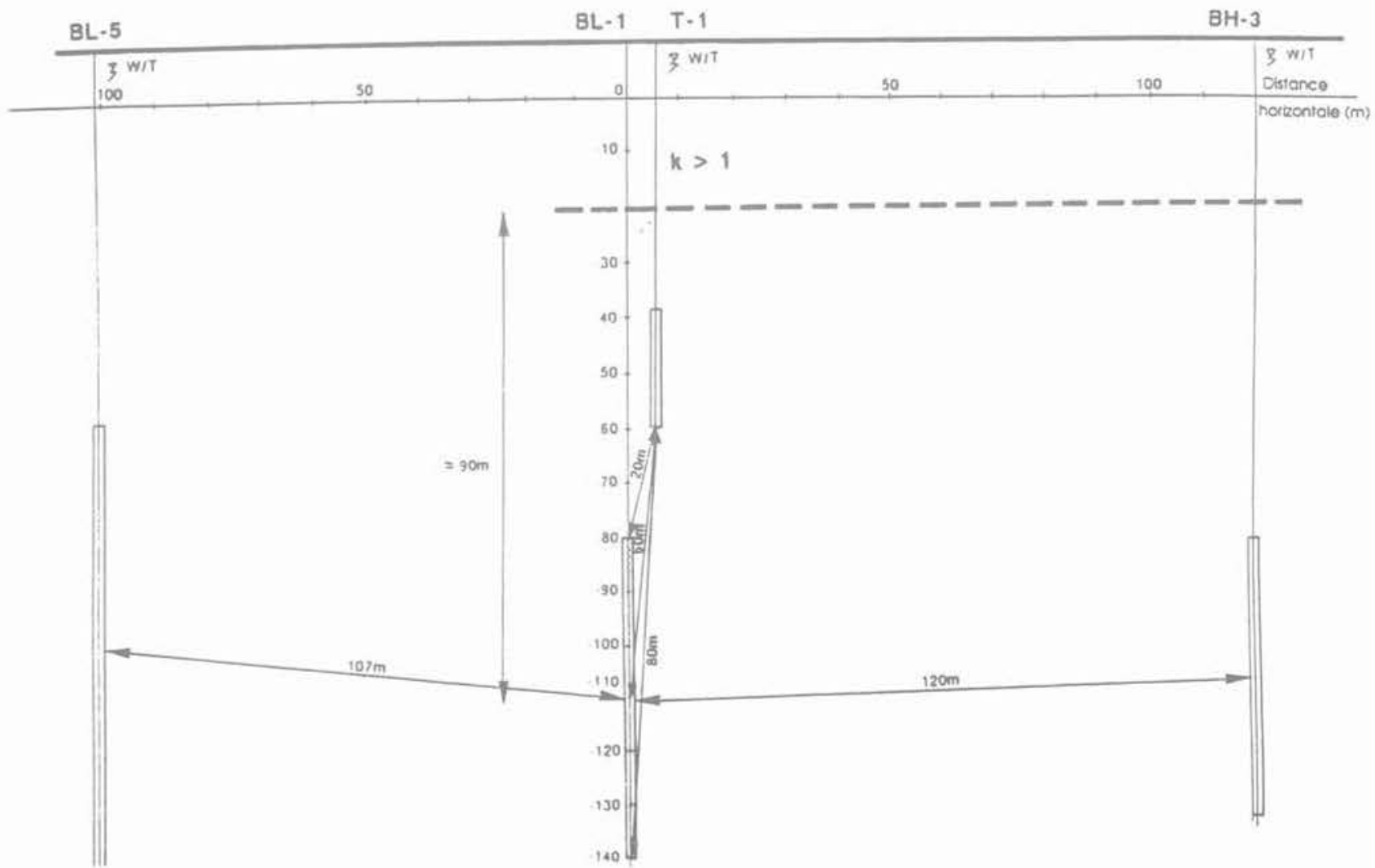

Fig. 1. - Test d'interférence de longue durée. Zone de BL-1. - Fig. 1. - Long duration interference test. BL-1 area. 
rieure à potentiel constant (existence d'une zone superficielle altérée plus fortement perméable).

\section{PRISE EN COMPTE DE L'ANISOTROPIE DANS LA CONCEPTION ET LE DIMENSIONNEMENT DES STOCKAGES EN CAVITÉS MINÉES}

\subsection{Théorie}

L'équation de la diffusivité hydraulique en régime permanent, en l'absence de source et en deux dimensions (ce qui est le cas d'une coupe verticale entre deux cavités) s'écrit :

$$
K x d^{2} h / d x^{2}+K y d^{2} h / d y^{2}=0
$$

avec :

$\mathrm{h}$ : potentiel hydraulique ;

$\mathrm{Kx}$ : perméabilité horizontale et Ky perméabilité verticale, si les axes $\mathrm{Ox}$ et $\mathrm{Oy}$ sont les deux directions principales d'anisotropie.

Si l'on fait apparaître le facteur $\mathrm{A}$ appelé coefficient d'anisotropie tel que:

$$
A=\sqrt{\mathrm{Ky} / \mathrm{Kx}}
$$

l'équation (1) devient :

$$
\mathrm{d}^{2} \mathrm{~h} / \mathrm{dx^{2 }}+\mathrm{Ky} / \mathrm{Kx} \mathrm{d}^{2} \mathrm{~h} / \mathrm{dy}^{2}=0
$$

Le problème anisotrope se ramène à un problème isotrope dans le nouveau système de coordonnées $\mathrm{x}^{\prime}$ et y' tels que :

$$
x^{\prime}=A x \text { et } y^{\prime}=y
$$

\subsection{Conséquences}

\section{sur la distance entre cavités}

La présence de cavités voisines est à l'origine d'interférences hydrauliques dans le milieu qui pourraient être pénalisantes du point de vue de l'exploitation en raison de la diminution des gradients hydrauliques, en particulier lorsque les écarts de potentiel sont importants. C'est le cas notamment lorsqu'une cavité est à la pression atmosphérique (phase de construction) et la cavité voisine à sa pression maximale. La définition de la distance critique dont dépendent les interférences entre cavités est donc essentielle pour le dimensionnement d'un projet.

D'après l'équation (2), on constate que le rapport d'anisotropie $(\mathrm{Kx} / \mathrm{Ky})$ influe directement sur la distance, ce qui implique les constatations suivantes:

- en cas de composante horizontale plus forte ( $\mathrm{Kx}$ $>\mathrm{Ky}$ ), la distance géométrique réelle $\mathrm{D}$ entre cavités sera équivalente sur le plan hydraulique à une distance fictive (Df) plus faible et telle que $\mathrm{Df}=\mathrm{D}$ $\sqrt{(\mathrm{Ky} / \mathrm{Kx})}$. L'effet de l'anisotropie reviendra dans ce cas à rapprocher artificiellement les deux cavités et donc à accroître l'importance des phénomènes d'interférences. En l'absence de prise en compte des phé- nomènes d'anisotropie, l'éloignement des cavités d'une distance $\mathrm{D}$ théorique obtenue à l'aide d'un modèle homogène et isotrope conduirait, en pratique, à des limitations dans l'exploitation des cavités qui ne pourraient être exploitées indépendamment l'une de l'autre ;

- dans le cas contraire ( $\mathrm{Kx}<\mathrm{Ky}$ ), l'effet est favorable, ce qui minimise les interférences et permet de rapprocher les deux cavités d'où une exigence domaniale moindre.

Dans le cas où existeraient des contraintes d'implantation ne permettant pas d'éloigner les cavités autant que les calculs le montrent, il est alors nécessaire d'avoir recours à des remèdes artificiels. Un rideau d'eau vertical entre les deux unités est alors implanté. Son rôle est de constituer une condition limite verticale à potentiel hydraulique constant, minimisant ainsi les interférences éventuelles entre les stockages en assurant la sécurité hydraulique requise.

La détermination in situ du coefficient d'anisotropie est donc primordiale quant à Pimplantation de deux cavités voisines et au calcul de la distance critique à respecter dans un contexte hydrogéologique donné, d'autant plus que les dispositifs artificiels peuvent être sujets à évolution dans le temps (phénomènes néfastes de colmatage par exemple).

\subsection{Conséquences sur les consignes d'exploitation}

Certains cas d'anisotropie de perméabilité conduisent à adapter l'exploitation en fonction des contraintes hydrogéologiques. C'est le cas d'un contexte pour lequel une anisotropie de type $\mathrm{Kh} / \mathrm{Kv}=10$ est observée au niveau de la cote des galeries. Le test d'étanchéité final (phase de pressurisation à l'air de longue durée) nécessaire à la réception du stockage, a permis de mettre en évidence lexistence de phénomènes transitoires à proximité immédiate de la paroi. Ces phénomènes sont dus à une distorsion des courbes équipotentielles dont l'origine, dans ce cas particulier, est la présence de bancs peu perméables introduisant une hétérogénéité responsable de l'anisotropie.

La prise en compte de tels phénomènes impose en exploitation la définition d'une vitesse maximale admissible de montée en pression de la cavité, et le respect strict de ces règles en cours d'exploitation.

Dans le cas où la prise en compte d'un contraste d'anisotropié défavorable entraînerait des contraintes d'exploitation trop sévères, il serait alors nécessaire d'avoir recours à des remèdes du type rideau d'eau par exemple.

\section{CONCLUSION}

Les investigations hydrogéologiques menées par GÉOSTOCK lors de la reconnaissance de site en vue du stockage de GPL en cavité minée ont permis de rencontrer une diverstité de configurations d'anisotropie de distribution de perméabilités. La mesure du coefficient d'anisotropie est essentielle pour le dimen- 
sionnement des cavités (pressions de fonctionnement, distance critique entre unités, règles d'exploitation) et nécessite donc des moyens d'investigation hydrogéologiques appropriés et adaptés à l'échelle du projet.

\section{BIBLIOGRAPHIE}

BEREST P. et al. (1983), Etanchéité des stockages souterrains de GPL en galeries non couvertes.
5 th int. Congress on Rock Mechanics, Melbourne.

FONTAN G. et AMANTINI E. (1989), Le confinement des gaz stockés en cavités minées par leur environnement hydrogéologique. Symp. on Current development and trends in underground storage of natural gas and LPG, Paris.

FONTAN G., Underground storage and environmental protection. Int. symp. Rockstore, Sweden. 